Article

\title{
Three-Phase Harmonic Analysis Method for Unbalanced Distribution Systems
}

\author{
Jen-Hao Teng ${ }^{1, *}$, Shu-Hung Liao ${ }^{1}$ and Rong-Ceng Leou ${ }^{2}$ \\ 1 Department of Electrical Engineering, National Sun Yat-Sen University, Kaohsiung 80424, Taiwan; \\ E-Mail: lunaguard@hotmail.com \\ 2 Department of Electrical Engineering, Cheng-Shiu University, Kaohsiung 83347, Taiwan; \\ E-Mail: leou@csu.edu.tw \\ * Author to whom correspondence should be addressed; E-Mail: jhteng@ee.nsysu.edu.tw; \\ Tel.: +886-7-5252000 (ext. 4118); Fax: +886-7-5254199.
}

Received: 8 November 2013; in revised form: 18 December 2013 / Accepted: 9 January 2014 / Published: 20 January 2014

\begin{abstract}
Due to the unbalanced features of distribution systems, a three-phase harmonic analysis method is essential to accurately analyze the harmonic impact on distribution systems. Moreover, harmonic analysis is the basic tool for harmonic filter design and harmonic resonance mitigation; therefore, the computational performance should also be efficient. An accurate and efficient three-phase harmonic analysis method for unbalanced distribution systems is proposed in this paper. The variations of bus voltages, bus current injections and branch currents affected by harmonic current injections can be analyzed by two relationship matrices developed from the topological characteristics of distribution systems. Some useful formulas are then derived to solve the three-phase harmonic propagation problem. After the harmonic propagation for each harmonic order is calculated, the total harmonic distortion (THD) for bus voltages can be calculated accordingly. The proposed method has better computational performance, since the time-consuming full admittance matrix inverse employed by the commonly-used harmonic analysis methods is not necessary in the solution procedure. In addition, the proposed method can provide novel viewpoints in calculating the branch currents and bus voltages under harmonic pollution which are vital for harmonic filter design. Test results demonstrate the effectiveness and efficiency of the proposed method.
\end{abstract}

Keywords: unbalanced distribution system; harmonic analysis; harmonic filter design; THD 


\section{Introduction}

Due to the increasing usage of nonlinear loads, harmonics pollution has became a growing problem in distribution systems. Many methods have been proposed to analyze and eliminate the harmonic impact produced by nonlinear loads [1-16]. Among them, harmonic analysis is one of the most powerful tools for harmonic impact analysis. It can be used to quantify the harmonic distortion for power systems and to determine whether dangerous resonant problems exist. Commonly-used harmonic analysis methods can be divided into two categories [1-12]. The first category is based on the techniques of time-domain analysis, wavelet analysis, etc. [1-5]. Time-domain-based methods are very flexible, but it is difficult to consider nonlinear loads in terms of specified power and they often lead to long simulation times. The second category is based on the techniques of steady-state analysis, which has better computational performance and therefore is suitable for large-scale power system analysis [1-4,6-12]. Steady-state-based methods are mostly developed based on load flow programs or admittance matrix inverses and employ frequency-based component models.

Traditional harmonic analysis methods are developed based on single-phase representation, and they cannot be used straightforwardly in distribution systems due to the topological characteristics of distribution systems. The well-known topological characteristics of distribution systems include radial network structure, unbalanced loads, multi-phase and unbalanced operation, large number of branches and nodes, wide-ranging resistance and reactance values, etc. Owing to the unbalanced line sections and loads in distribution systems, a three-phase harmonic analysis method is essential to accurately analyze the harmonic impact on distribution systems [7-12]. The harmonic analysis is also the basic tool for harmonic filter design and harmonic resonance mitigation and has to be done repeatedly for each harmonic order [13-16]; therefore, the accuracy and efficiency are both important issues for a well-designed harmonic analysis method. Conventional harmonic analysis methods [7-11] don't consider the special topological characteristics of distribution systems such as the unbalanced loads and radial network structure; therefore, the solution accuracy cannot be further improved and computational time cannot be effectively reduced. A harmonic analysis method was proposed based on frequency-scan formulation, exact three-phase models, and commonly used forward/backward sweep technique that can fully use the topological characteristics of distribution systems [12]. However, some specific data formats, such as the coefficient vector defined to record whether the system harmonic currents flow through the branch, needed to be designed and integrated into the commonly used forward/backward sweep technique. Therefore, the method proposed in [12] cannot be used straightforwardly to solve other harmonic problems, i.e., optimal harmonic filter design, etc.

An accurate and efficient three-phase harmonic analysis method for unbalanced distribution systems is proposed in this paper. The variations of bus voltages, bus current injections and branch currents affected by harmonic currents of nonlinear loads and shunt equipments can be analyzed by two relationship matrices developed from the topological characteristics of distribution systems. Therefore, the topological characteristics of distribution systems can be completely integrated into the proposed method for computational performance improvement. Some useful formulas are derived to solve the three-phase harmonic propagation problem. After the harmonic propagation for each harmonic order was calculated, the total harmonic distortion (THD) for bus voltages could be calculated accordingly. Exact three-phase models are used for the proposed harmonic analysis method; therefore, accurate 
solutions can be obtained. The proposed method is also efficient, since the time-consuming full admittance matrix inverse employed by commonly-used harmonic analysis methods is not necessary in the solution procedure. Compared with the method proposed in [12], no specific data format needs to be designed and integrated in the proposed solution procedure. Moreover, the proposed method can provide novel viewpoints in calculating the branch currents and bus voltages under harmonic pollution. The harmonic currents aborted by the shunt equipments such as harmonic filters can also be easily quantified in the proposed solution procedure. This data is vital for harmonic filter design and harmonic resonance mitigation. Test results demonstrate the validity of the proposed method.

\section{Basic Concepts}

\subsection{Commonly-Used Harmonic Analysis Method}

Due to the unbalanced line sections and loads in distribution systems, three-phase models should be used in harmonic analysis. For example, the impedance matrix of a three-phase line section ([ $\left.\left.\mathbf{Z}^{a b c}\right]\right)$ can be expressed as:

$$
\left[\mathbf{Z}^{a b c}\right]=\left[\begin{array}{lll}
Z^{a a} & Z^{a b} & Z^{a c} \\
Z^{b a} & Z^{b b} & Z^{b c} \\
Z^{c a} & Z^{c b} & Z^{c c}
\end{array}\right]
$$

The commonly-used harmonic analysis method [1,6] is developed based on the full admittance matrix inverse of a distribution system and can be obtained by:

$$
\left[\mathbf{Y}^{(h)}\right]\left[\mathbf{V}^{(h)}\right]=\left[\mathbf{I}^{(h)}\right]
$$

where $\left[\mathbf{Y}^{(h)}\right]$ is the full admittance matrix of a distribution system for the $h^{\text {th }}$-order harmonic; $\left[\mathbf{V}^{(h)}\right]$ and $\left[\mathbf{I}^{(h)}\right]$ are the vectors of bus voltage and current injection for the $h^{\text {th }}$-order harmonic, respectively.

Equation (2) can be solved by triangular factorization and forward/backward substitution or by an inverse matrix. The procedures are time-consuming for large-scale unbalanced distribution systems. Especially, a harmonic propagation calculation should be done repeatedly for each harmonic order. If the topological characteristics of distribution systems can be taken into account to avoid the triangular factorization of a full admittance matrix, computational time can be significantly reduced. The voltage THD after the harmonic propagation of each order has been solved can be represented as:

$$
\operatorname{VTHD}_{i}(\%)=\frac{\sqrt{\sum_{h=2}^{n}\left|V_{i}^{(h)}\right|^{2}}}{\left|V_{i}^{(1)}\right|} \times 100 \%
$$

where $V_{i}^{(h)}$ and $V T H D_{i}(\%)$ are the $h^{\text {th }}$-order harmonic voltage and voltage THD percentage for bus $i$, respectively.

\subsection{Proposed Relationship Matrices}

The proposed method is developed based on two relationship matrices, the bus-current-injection to branch-current (BIBC) matrix and branch-current to bus-voltage (BCBV) matrix. Detailed derivations 
of these two matrices can be found in $[17,18]$. In this paper, only the building algorithms of these two matrices are shown. The relationship between bus current injections and branch currents for a radial distribution system can be expressed as follows:

$$
[\mathbf{B}]=[\mathbf{B I B C}][\mathbf{I}]
$$

where $[\mathbf{B I B C}]$ is the bus-current-injection to branch-current matrix for a radial distribution system; $[\mathbf{I}]$ and $[\mathbf{B}]$ are the vectors for bus current injections and branch currents, respectively.

The constant BIBC matrix is an upper triangular matrix and has non-zero entries of +1 only. The building algorithm of BIBC matrix from substation to downstream sequentially is:

Procedure 1 For a distribution system with $m$-branch sections and $n$-bus, the dimension of the BIBC matrix is $m \times(n-1)$;

Procedure 2 If a line section $\left(B_{k}\right)$ is located between bus $i$ and bus $j$, copy the column of the $i$-th bus of the BIBC matrix to the column of the $j$-th bus and fill $\mathrm{a}+1$ to the position of the $k$-th row and $j$-th bus column;

Procedure 3 Repeat Procedure 2 until all line sections are included in the BIBC matrix.

The relationship between branch currents and bus voltages for a radial distribution system can be written as:

$$
[\Delta \mathbf{V}]=\left[\mathbf{V}_{0}\right]-[\mathbf{V}]=[\mathbf{B C B V}][\mathbf{B}]
$$

where $[\mathbf{B C B V}]$ is the branch-current to bus-voltage matrix; $\left[\mathbf{V}_{0}\right]$ and $[\mathbf{V}]$ are the vectors of no-load bus voltages and bus voltages for distribution systems, respectively. For a distribution system with $N$ buses, the dimension for $\left[\mathbf{V}_{0}\right]$ and $[\mathbf{V}]$ is $(N-1)$ by 1 .

The constant BCBV matrix has non-zero entries consisted of complex line impedance values. The building algorithm of $\mathbf{B C B V}$ matrix from substation to downstream sequentially is:

Procedure 4 For a distribution system with $m$-branch sections and $n$-bus, the dimension of the BCBV matrix is $(n-1) \times m$;

Procedure 5 If a line section $\left(B_{k}\right)$ is located between bus $i$ and bus $j$, copy the row of the $i$-th bus of the BCBV matrix to the row of the $j$-th bus and fill the line impedance $\left(Z_{i j}\right)$ to the position of the $j$-th bus row and $k$-th column;

Procedure 6 Repeat Procedure 5 until all line sections are included in the BCBV matrix.

Note that if the line section between bus $i$ and bus $j$ as represented in Procedures 1-6 is a three-phase line section, the corresponding branch current $B_{k}$ will be a $3 \times 1$ vector, the $+1 \mathrm{~s}$ in the BIBC matrix become a $3 \times 3$ identity matrix, and the $Z_{i j}$ in the $\mathbf{B C B V}$ matrix constitute a $3 \times 3$ impedance matrix. Equations (4) and (5) are very useful for derivations of the proposed harmonic analysis method. Detailed formulas will be derived in the next section.

\section{Three-Phase Harmonic Analysis Method}

Equations (4) and (5) provide a new perspective in observing the relationship between bus voltages, bus current injections and branch currents. As can be seen in Equation (4), the mathematical equation between bus current injections and branch currents, can be used to calculate the variations of the 
branch currents affected by the harmonic current injections. In Equation (5), the mathematical equation between the branch currents and bus voltages, can be used to calculate the variations of the bus voltages resulting from the harmonic branch currents. Figure 1 illustrates the concepts of the proposed harmonic propagation. The harmonic currents flowing in a distribution system can be divided into two categories: the harmonic currents contributed by nonlinear loads, $I s^{(h)}$ as denoted in Figure 1, and the harmonic currents absorbed by shunt equipments, $I f^{(h)}$ as denoted in Figure 1, such as harmonic filters. In general, harmonic currents contributed by nonlinear loads can be obtained by field measurements and/or mathematical formulas. Some research aimed to find the mathematic formulas for the harmonic currents contributed by nonlinear loads [7,19]. Using the derived mathematical formulas, the harmonic impacts of nonlinear loads can be conducted only by simulations; therefore, the cost and time for power quality analyzer installation and measurement can be saved. The harmonic currents obtained by mathematical formulas can also be used in the proposed harmonic analysis method. The harmonic currents absorbed by shunt equipments need to be calculated according to the network topology and harmonic currents of nonlinear loads. If the harmonic currents absorbed by shunt equipments were calculated, the branch currents and bus voltages affected by these two types of harmonic currents could then be analyzed by Equations (4) and (5), respectively.

Figure 1. Concepts of the proposed harmonic propagation.

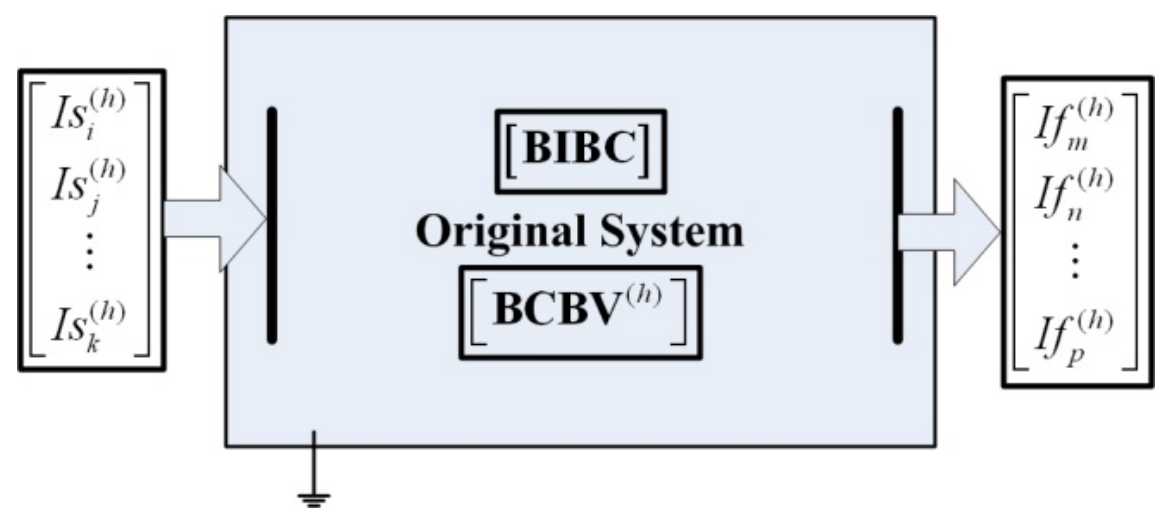

The harmonic currents of nonlinear loads can be expressed in a vector form as:

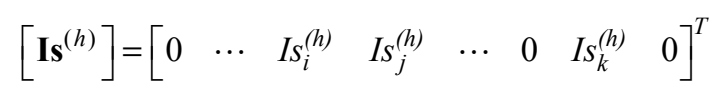

where $\left[\mathbf{I} \mathbf{s}^{(h)}\right]$ is the $h^{\text {th }}$-order harmonic current vector of nonlinear loads.

The branch currents affected by the $h^{\text {th }}$-order harmonic currents of nonlinear loads can be calculated by substituting Equation (6a) into Equation (4) and be expressed as:

$$
\left[\mathbf{B s}^{(h)}\right]=[\mathbf{B I B C}]\left[\mathbf{I s}^{(h)}\right]
$$

where $\left[\mathbf{B s}^{(h)}\right]$ is the branch current vector affected by the $h^{\text {th }}$-order harmonic currents of nonlinear loads.

Similarly, the $h^{\text {th }}$-order harmonic current vectors for shunt equipments, [If $\left.{ }^{(h)}\right]$, and branch currents affected by the $h^{\text {th }}$-order harmonic currents of shunt equipments, $\left[\mathbf{B f}{ }^{(h)}\right]$, can be expressed as:

$$
\begin{aligned}
& {\left[\mathbf{I f}^{(h)}\right]=\left[\begin{array}{llllllll}
0 & \cdots & I f_{m}^{(h)} & I f_{n}^{(h)} & \cdots & 0 & I f_{o}^{(h)} & 0
\end{array}\right]^{T}} \\
& {\left[\mathbf{B f}^{(h)}\right]=[\mathbf{B I B C}]\left[\mathbf{I f}^{(h)}\right]}
\end{aligned}
$$


The aggregate branch current vector affected by the $h^{\text {th }}$-order harmonic currents of nonlinear loads and shunt equipments, $\left[\mathbf{B}^{(h)}\right]$, can be written as:

$$
\left[\mathbf{B}^{(h)}\right]=\left[\mathbf{B s}^{(h)}\right]+\left[\mathbf{B f}^{(h)}\right]
$$

Equation (8) can be rewritten as:

$$
\begin{aligned}
& {\left[\mathbf{B}^{(h)}\right]=\sum_{g=1}^{N s}\left[\mathbf{B I B C}_{x}\right] I s_{x}^{(h)}+\sum_{h=1}^{N f}\left[\mathbf{B I B C}_{y}\right] I f_{y}^{(h)}} \\
& x=\operatorname{Isbus}(g) ; y=\operatorname{Ifbus}(h)
\end{aligned}
$$

where $\operatorname{ISbus}(\cdot)$ and $\operatorname{Ifbus}(\cdot)$ are the buses relating to the nonlinear loads and shunt equipments, respectively. $N s$ and $N f$ are the numbers of nonlinear loads and shunt equipments, respectively. $\left[\mathbf{B I B C}_{z}\right]$ is the column vector of $[\mathbf{B I B C}]$ relating to the bus of $I s_{z}^{(h)}$ or $I f_{z}^{(h)}$.

The bus voltages affected by the harmonic branch currents can be obtained by substituting Equation (9) into Equation (5) and be represented as:

$$
\left[\Delta \mathbf{V}^{(h)}\right]=\left[\mathbf{B C B} \mathbf{V}^{(h)}\right]\left[\mathbf{B}^{(h)}\right]
$$

Equation (10) can be further expressed as:

$$
\left[\Delta \mathbf{V}^{(h)}\right]=\left[\mathbf{B C B V} \mathbf{V}^{(h)}\right]\left(\sum_{g=1}^{N s}\left[\mathbf{B I B C}_{x}\right] I s_{x}^{(h)}+\sum_{h=1}^{N f}\left[\mathbf{B I B C}_{y}\right] I f_{y}^{(h)}\right)
$$

The voltage of bus $i$ in Equation (11) can therefore be rewritten as:

$$
\Delta V_{i}^{(h)}=\left[\mathbf{B C B V}_{i}^{(h)}\right]\left(\sum_{g=1}^{N s}\left[\mathbf{B I B C}_{x}\right] I s_{x}^{(h)}+\sum_{h=1}^{N f}\left[\mathbf{B I B C}_{y}\right] I f_{y}^{(h)}\right)
$$

where $\left[\mathbf{B C B V}_{i}\right]$ is the row vector of $[\mathbf{B C B V}]$ relating to bus $i$.

The voltages of the shunt equipments such as harmonic filters can also be calculated by multiplying their harmonic currents absorbed and harmonic impedance. For example, the voltage of shunt equipment at bus $y$ can be expressed as:

$$
\Delta V_{y}^{(h)}=-Z f_{y}^{(h)} \times I f_{y}^{(h)}
$$

where $V_{y}^{(h)}$ and $Z f_{y}^{(h)}$ are the bus voltage and shunt impedance of the $h^{\text {th }}$-order harmonic at bus $y$, respectively.

The bus voltages of shunt equipments can then be expressed in vector form as Equation (14). Equation (14) can be rearranged and written as Equation (15) and then Equation (16) can be derived. Therefore, the harmonic currents absorbed by the shunt equipments can be calculated by Equation (17). After the harmonic currents absorbed by shunt equipments are calculated, the branch currents and bus voltages affected by the $h^{\text {th }}$-order harmonic currents can be calculated by Equations (9) and (11), respectively. The main equipment that needs to be considered in the distribution harmonic analysis includes transformers, capacitors, inductors and line sections:

$$
-\left[\begin{array}{c}
Z f_{I f b u s(1)}^{(h)} I f_{I f b u s(1)}^{(h)} \\
\vdots \\
Z f_{\text {Ifbus }(N f)}^{(h)} I f_{\text {Ifbus }(N f)}^{(h)}
\end{array}\right]=\left[\begin{array}{c}
\mathbf{B C B} \mathbf{V}_{\text {Ifbus }(1)}^{(h)} \\
\vdots \\
\mathbf{B C B V}_{\text {Ifbus }(N f)}^{(h)}
\end{array}\right] \times\left(\sum_{g=1}^{N s}\left[\mathbf{B I B C}_{x}\right] I s_{x}^{(h)}+\sum_{h=1}^{N f}\left[\mathbf{B} \mathbf{B} \mathbf{B C}_{y}\right] I f_{y}^{(h)}\right)
$$




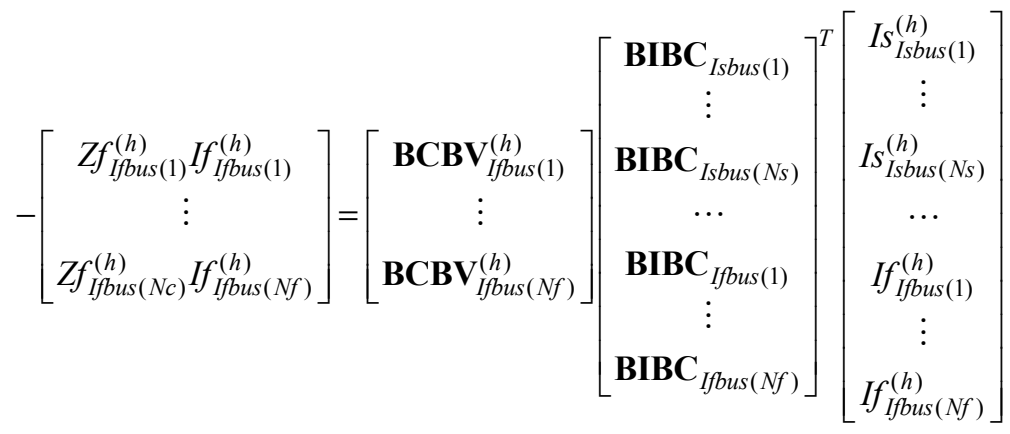

$$
\begin{aligned}
& -\left[\mathbf{Z F}^{(h)}\right]\left[\begin{array}{c}
I f_{\text {Ifbus }(1)}^{(h)} \\
\vdots \\
I f_{\text {Ifbus }(N f)}^{(h)}
\end{array}\right]=\left[\mathbf{Z I S}^{(h)}\right]\left[\begin{array}{c}
I S_{\text {Isbus (1) }}^{(h)} \\
\vdots \\
I S_{\text {Isbus }(N s)}^{(h)}
\end{array}\right]+\left[\mathbf{Z I F}^{(h)}\right]\left[\begin{array}{c}
I f_{\text {Ifbus }(1)}^{(h)} \\
\vdots \\
I f_{\text {Ifbus }(N f)}^{(h)}
\end{array}\right] \\
& {[\mathbf{Z F}]=\left[\begin{array}{ccc}
Z f_{I f b u s(1)}^{(h)} & 0 & \cdots \\
0 & \ddots & 0 \\
\vdots & 0 & Z f_{\text {Ifbus }(N f)}^{(h)}
\end{array}\right]} \\
& {[\mathbf{Z I S}]=\left[\begin{array}{c}
\mathbf{B C B V}_{\text {Ifbus }(1)}^{(h)} \\
\vdots \\
\mathbf{B C B V}_{\text {Ifbus }(N f)}^{(h)}
\end{array}\right]\left[\begin{array}{c}
\mathbf{B I B C}_{I s b u s(1)} \\
\vdots \\
\mathbf{B I B C}_{\text {Isbus }(N s)}
\end{array}\right]^{T}} \\
& {[\mathbf{Z I F}]=\left[\begin{array}{c}
\mathbf{B C B V}_{\text {Ifbus }(1)}^{(h)} \\
\vdots \\
\mathbf{B C B V}_{\text {Ifbus }(N f)}^{(h)}
\end{array}\right]\left[\begin{array}{c}
\mathbf{B I B C}_{I f f u s(1)} \\
\vdots \\
\mathbf{B I B C}_{I f b u s(N f)}
\end{array}\right]^{T}} \\
& {\left[\begin{array}{c}
I f_{\text {Ifbus }(1)}^{(h)} \\
\vdots \\
I f_{\text {Ifbus }(N f)}^{(h)}
\end{array}\right]=-\left[\mathbf{Z H A}^{(h)}\right]^{-1}\left[\mathbf{Z I S}^{(h)}\right]\left[\begin{array}{c}
I S_{\text {Isbus }(1)}^{(h)} \\
\vdots \\
I S_{\text {Isbus }(N)}^{(h)}
\end{array}\right]} \\
& {\left[\mathbf{Z H A}{ }^{(h)}\right]=\left[\mathbf{Z I F}^{(h)}+\mathbf{Z F} \mathbf{F}^{(h)}\right]}
\end{aligned}
$$

From Equation (17), it can be illustrated that for a distribution system with $N f$ shunt equipment, $\left[\right.$ ZHA $\left.^{(h)}\right]$ is a square matrix with dimension $N f$. Instead of inverting the full admittance matrix of a distribution system, only the matrix with dimension $N f$ needs to be inverted; therefore, the proposed method has better computational performance. Besides, the proposed method can provide novel viewpoints in calculating the branch currents and bus voltages under harmonic pollution.

In general, a swing bus should be assigned for load flow calculation. For distribution systems, the swing bus is the substation bus and its voltage will maintain constant under all conditions. In harmonic analysis, the harmonic propagation for the substation bus can also be calculated if necessary. Namely, the harmonic currents can propagate through the distribution substation and beyond. In this situation, the short-circuit capacity in a substation bus can be used to integrate the substation bus into harmonic analysis. The strength of the substation bus is directly proportional to its short-circuit capacity. The Thevenin equivalent impedance looking back into the transmission system from the substation bus can be calculated by its short-circuit capacity [20]. The Thevenin equivalent impedance in p.u. can be expressed as: 


$$
Z_{t h}^{S / S}=\frac{M V A_{B}}{M V A_{S C}}
$$

where $Z_{t h}^{S / S}$ is the Thevenin equivalent impedance of the substation bus with respect to the short-circuit capacity $\left(M V A_{S C}\right)$ and base MVA $\left(M V A_{B}\right)$.

Figure 2 illustrates the concepts of including the substation bus into harmonic analysis, and it can be seen that the substation bus can be treated as the Thevenin equivalent impedance of its short-circuit capacity. The transmission system can then be considered as an infinite bus with infinite short-circuit capacity and zero equivalent impedance and will maintain its voltage under all conditions. Therefore, the substation bus can be integrated into the proposed method without difficulty.

Figure 2. Including the substation bus in harmonic analysis.

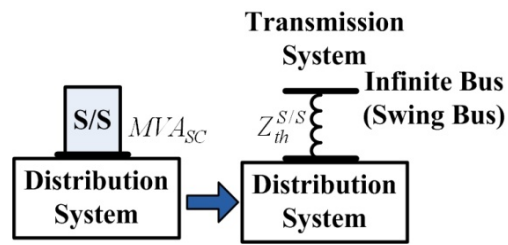

The frequency-based component models as presented in $[2-4,6]$ are used for the proposed harmonic analysis method. In a distribution system, the main devices considered in the harmonic analysis include distribution cable, transformer, capacitor, inductor, etc. Using the frequency-based component models, the line impedances required for the BCBV matrix under different harmonic orders are scalable. The BIBC matrix is a constant matrix and will not need to be modified in the proposed harmonic analysis method. A large-scale induction motor can be treated as a harmonic source with impedance. Therefore, the frequency-based component models can also be utilized. Other models can also be integrated into the proposed method if the relationship between the component impedance and harmonic frequency can be obtained.

\section{Test Results and Discussions}

Many distribution systems have been tested; however, only several cases are shown here to explain the solution procedures and demonstrate the validity of the proposed method. The fundamental frequency and base voltage for the following test cases are $60 \mathrm{~Hz}$ and $11.4 \mathrm{kV}$, respectively.

\subsection{Simple Seven-Bus System}

A simple seven-bus system as shown in Figure 3 is used as an example to build the matrices and vectors in Equation (16). The simple seven-bus system (equivalent to an 18-node system) consists of the three-phase, double-phase and single-phase line sections and buses. The simple seven-bus system and the nonlinear loads are not based on a real-world situation and are only used to explain the solution procedures of the proposed harmonic analysis method. Besides, for explanation purposes, many harmonic filters are added to the simple seven-bus system. Figure 3 illustrates that nonlinear loads and harmonic filters are interconnected to Buses 3 and 6, respectively. The matrices and vectors used for the proposed method are shown in the Appendix. To simplify the case explained in the Appendix, only the situation of nonlinear loads in Bus 3 as illustrated in Figure 3 is tested. 
Figure 3. A simple seven-bus test system.

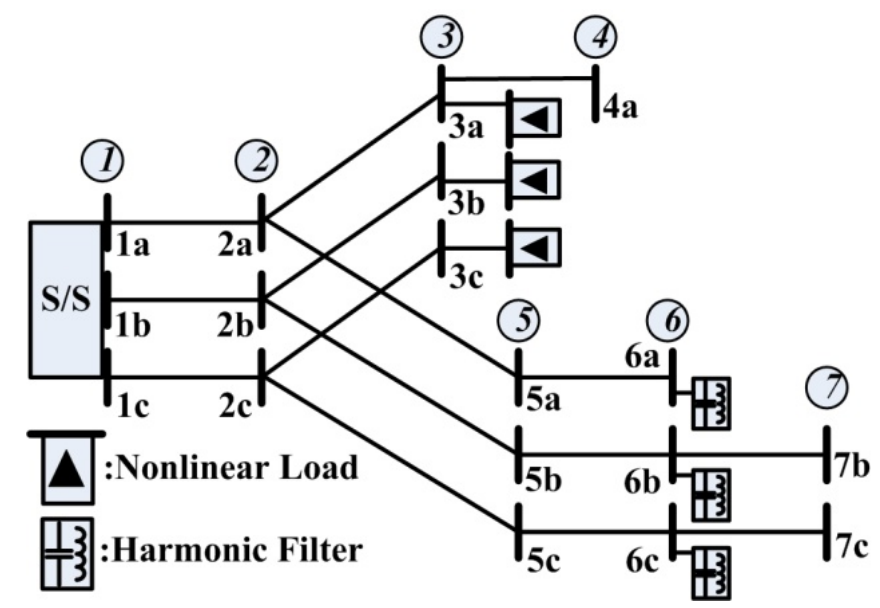

Nonlinear loads in Buses 3 and 5 as illustrated in Figures 4-7 are simulated in the numerical cases.

Figure 4. Branch currents of the $5^{\text {th }}$-order harmonic order.



Figure 5. Bus voltage of the $5^{\text {th }}$-order harmonic order.




Figure 6. Branch currents of the $5^{\text {th }}$-order harmonic order after harmonic filter parameter adjustment.

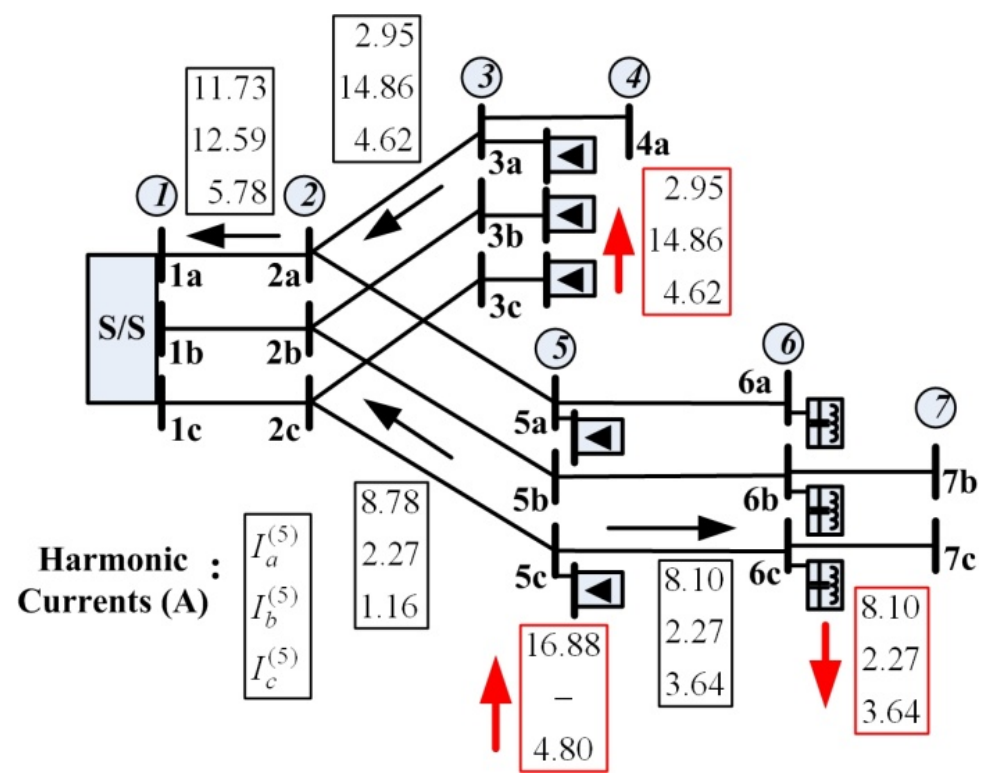

Figure 7. Bus voltage of the $5^{\text {th }}$-order harmonic order with substation bus.

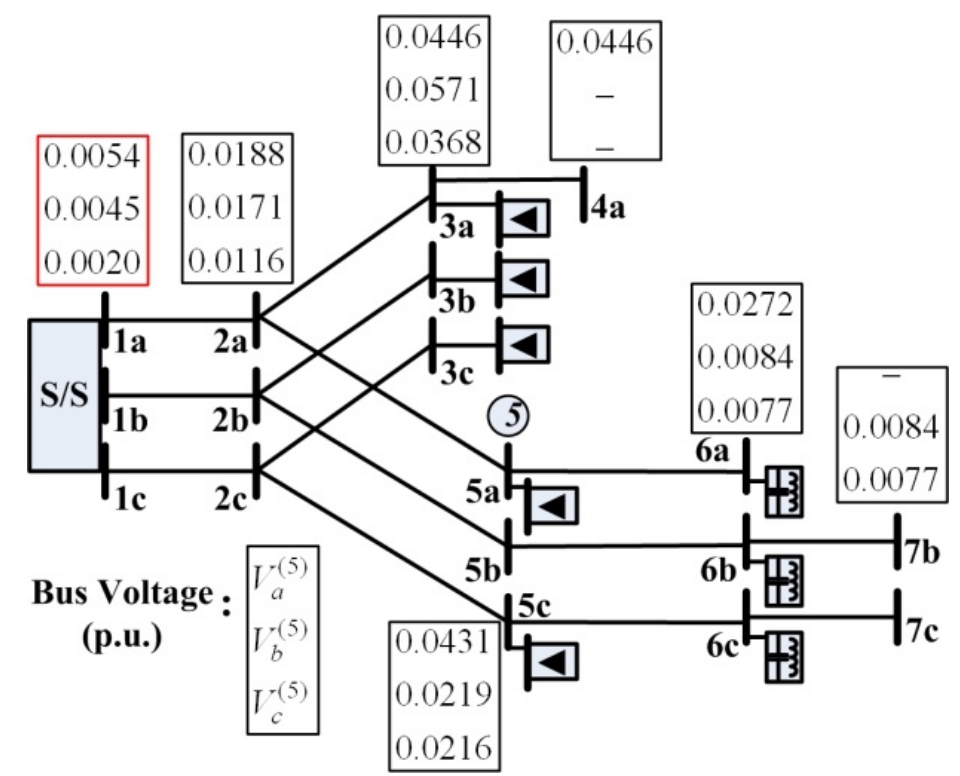

Tables 1 and 2 show the line data and bus data for the simple seven-bus system of Figure 3, respectively.

Table 1. Line data of the simple seven-bus test system.

\begin{tabular}{ccccc}
\hline Line number & From bus & End bus & Phases & Line length (miles) \\
\hline 1 & 1 & 2 & $a b c$ & 0.8 \\
2 & 2 & 3 & $a b c$ & 2.8 \\
3 & 3 & 4 & $a$ & 4.3 \\
4 & 2 & 5 & $a b c$ & 3.3 \\
5 & 5 & 6 & $a b c$ & 2.2 \\
6 & 5 & 7 & $b c$ & 3.8 \\
\hline
\end{tabular}


Table 2. Load data of a simple seven-bus test system.

\begin{tabular}{ccccccccc}
\hline \multirow{2}{*}{ Bus } & \multicolumn{2}{c}{ Phase $\boldsymbol{a}$} & & \multicolumn{2}{c}{ Phase $\boldsymbol{b}$} & & \multicolumn{2}{c}{ Phase $\boldsymbol{c}$} \\
\cline { 2 - 3 } \cline { 7 - 8 } \cline { 7 - 8 } & $\mathbf{k W}$ & $\mathbf{k V A R}$ & & $\mathbf{k W}$ & $\mathbf{k V A R}$ & & $\mathbf{k W}$ & $\mathbf{k V A R}$ \\
\hline 1 & - & - & & - & - & & - & - \\
2 & 360 & 70 & & 432 & 50 & & 120 & 30 \\
3 & 145 & 10 & & 643 & 100 & & 445 & 76 \\
4 & 145 & 28 & & - & - & & - & - \\
5 & 732 & 112 & & 345 & 98 & & 225 & 63 \\
6 & 432 & 82 & & 377 & 93 & & 425 & 103 \\
7 & - & - & & 632 & 312 & & 645 & 198 \\
\hline
\end{tabular}

The three-phase, double-phase and single-phase line parameters are expressed in Equations (19a)-(19c), respectively:

$$
\begin{gathered}
{\left[\mathbf{Z}^{a b c}\right](\Omega / m i)=\left[\begin{array}{ccc}
0.3477+j 1.0141 & 0.1565+j 0.4777 & 0.1586+0.4361 \\
0.1565+j 0.4777 & 0.3375+j 1.0478 & 0.1535+j 0.3849 \\
0.1586+0.4361 & 0.1535+j 0.3849 & 0.3414+j 1.0348
\end{array}\right]} \\
{\left[\mathbf{Z}^{b c}\right](\Omega / m i)=\left[\begin{array}{ll}
0.3477+j 1.0141 & 0.1565+j 0.4777 \\
0.1565+j 0.4777 & 0.3375+j 1.0478
\end{array}\right]} \\
{\left[\mathbf{Z}^{a}\right](\Omega / m i)=[0.3477+j 1.0141]}
\end{gathered}
$$

Table 3 lists the bus voltages solved by the unbalanced distribution load flow method proposed in [17], and it can be seen that the feeder is operated under unbalanced conditions.

Table 3. Load flow solution of the simple seven-bus test system.

\begin{tabular}{ccccccccc}
\hline \multirow{2}{*}{ Bus } & \multicolumn{2}{c}{ Phase $\boldsymbol{a}$} & & \multicolumn{2}{c}{ Phase $\boldsymbol{b}$} & & \multicolumn{2}{c}{ Phase $\boldsymbol{c}$} \\
\cline { 2 - 3 } \cline { 7 - 8 } \cline { 7 - 8 } & $|\mathbf{V}| \mathbf{( p . u . )}$ & Phasor (degree) & & $|\mathbf{V}|$ (p.u.) & Phasor (degree) & & $|\mathbf{V}|$ (p.u.) & Phasor (degree) \\
\hline 1 & 1.0000 & 0.00 & & 1.0000 & -120.00 & & 1.0000 & 120.00 \\
2 & 0.9962 & -0.23 & & 0.9940 & -120.55 & & 0.9964 & 119.59 \\
3 & 0.9939 & -0.2385 & & 0.9881 & -121.14 & & 0.9957 & 119.18 \\
4 & 0.9913 & -0.50 & & - & - & & - & - \\
5 & 0.9902 & -0.96 & & 0.9772 & -121.61 & & 0.9823 & 118.29 \\
6 & 0.9919 & -0.90 & & 0.9644 & -122.12 & & 0.9762 & 117.34 \\
7 & - & - & 0.9437 & -122.55 & & 0.9739 & 116.13 \\
\hline
\end{tabular}

The harmonic current magnitudes of nonlinear loads as illustrated in Figure 3 are listed in Table 4. Note that nonlinear loads are interconnected in Buses 3 and 5 for the following tests. The parameters of single tune harmonic filters including resonance frequency installed in Bus 6 are as listed in Table 5. Substituting the data from Tables 4 and 5 and Equation (19) into Equations (A1) and (A2) and Equations (16) and (17), the harmonic currents absorbed for each harmonic order can be calculated. For example, the $5^{\text {th }}$-order harmonic currents absorbed by the harmonic filters installed in Bus 6 are 5.49 A, 2.91 A and 4.28 A for phases $a, b$ and $c$, respectively. The branch currents and bus voltages for the $5^{\text {th }}$-order harmonic order as illustrated in Figures 4 and 5 can be calculated by Equation (9) and (11), 
respectively. From Figures 4 and 5, the effects of harmonic current injections on branch currents and bus voltages can be clearly observed.

Table 4. Harmonic currents of nonlinear loads.

\begin{tabular}{cccccc}
\hline \multirow{2}{*}{ Order } & \multicolumn{5}{c}{ Harmonic current (A) } \\
\cline { 2 - 6 } & Bus 3a & Bus 3b & Bus 3c & Bus 5a & Bus 5c \\
\hline 2 & 0.09 & 0.49 & 0.07 & 0.56 & 0.15 \\
3 & 1.64 & 2.00 & 5.11 & 2.27 & 2.68 \\
4 & 0.00 & 0.00 & 0.00 & 0.00 & 0.00 \\
5 & 2.95 & 14.86 & 4.62 & 16.88 & 4.80 \\
6 & 0.00 & 0.00 & 0.00 & 0.00 & 0.00 \\
7 & 1.11 & 4.54 & 0.85 & 5.16 & 1.80 \\
8 & 0.00 & 0.00 & 0.00 & 0.00 & 0.00 \\
9 & 0.13 & 0.14 & 0.39 & 0.16 & 0.21 \\
10 & 0.00 & 0.00 & 0.00 & 0.00 & 0.00 \\
11 & 0.14 & 0.28 & 0.34 & 0.32 & 0.22 \\
12 & 0.00 & 0.00 & 0.00 & 0.00 & 0.00 \\
13 & 0.08 & 0.06 & 0.24 & 0.07 & 0.13 \\
14 & 0.00 & 0.00 & 0.00 & 0.00 & 0.00 \\
15 & 0.066665 & 0.05003 & 0.20666 & 0.05681 & 0.10842 \\
\hline
\end{tabular}

Table 5. Parameters of installed harmonic filters.

\begin{tabular}{cccc}
\hline Bus & Reactance $(\Omega)$ & Capacitance $(\Omega)$ & Resonance frequency $(\mathbf{H z})$ \\
\hline 6a & 39.221 & 866.4 & 282 \\
6a & 39.776 & 1732.8 & 396 \\
6a & 15.717 & 1732.8 & 630 \\
6b & 19.611 & 433.2 & 282 \\
6b & 12.867 & 577.6 & 402 \\
6b & 7.7109 & 866.4 & 636 \\
6c & 25.069 & 577.6 & 288 \\
6c & 18.737 & 866.4 & 408 \\
6c & 15.135 & 1732.8 & 642 \\
\hline
\end{tabular}

After the bus voltages for each harmonic order are calculated, and the voltage THD (\%), as listed in Table 6, can be obtained. The proposed harmonic analysis method obtains the same solution as those obtained by $[6,12]$; therefore, the validity of the proposed can be demonstrated. Since the solutions of the proposed method and the conventional methods are same, the solutions aren't shown in the paper. From the simple example, it can be seen that the proposed method can be used to calculate the bus voltages for each harmonic order and the voltage THD (\%) effectively. 
Table 6. Voltage total harmonic distortion (THD) (\%) of the simple seven-bus test system.

\begin{tabular}{ccccc}
\hline \multirow{2}{*}{ Voltage THD(\%) of the simple seven-bus test system } & \multicolumn{3}{c}{ Voltage THD (\%) } \\
\cline { 2 - 4 } & Phase $\boldsymbol{a}$ & Phase $\boldsymbol{b}$ & Phase $\boldsymbol{c}$ \\
\hline 1 & - & - & - \\
2 & 1.5548 & 1.4741 & 1.1856 \\
4 & 4.4592 & 5.9152 & 4.0361 \\
5 & 4.4711 & - & - \\
6 & 4.6002 & 2.5575 & 2.8233 \\
7 & 3.2839 & 2.0253 & 2.1593 \\
\hline
\end{tabular}

If the reactance and capacitance parameters of a harmonic filter installed in bus $6 \mathrm{a}$ with a resonance frequency $282 \mathrm{~Hz}$ are changed to $36.8331 \Omega$ and $866.4 \Omega$ (resonance frequency $291 \mathrm{~Hz}$ ), respectively, the $5^{\text {th }}$-order harmonic currents absorbed by the harmonic filters installed in Bus 6 will be changed to 8.103 A, 2.268 A and 3.641 A for phases $a, b$ and $c$, respectively. The branch currents after harmonic filter parameter adjustment are as shown in Figure 6. The effects of harmonic filter parameter adjustment on branch currents and bus voltages can therefore be effectively determined. The proposed method can provide novel viewpoints in calculating the branch currents and bus voltages under harmonic pollution. These novel viewpoints have great potential to be used for optimal harmonic filter design and harmonic resonance mitigation. Due to limited space, the applications will be investigated in future work.

If the substation bus with short-circuit capacity 250 MVA is included in the harmonic analysis, the bus voltages for the $5^{\text {th }}$-order harmonic order can then be calculated and illustrated in Figure 7 . The voltage THD (\%) of each bus is listed in Table 7. Note that the Thevenin equivalent impedance is 0.0004 p.u. with respect to the short-circuit capacity 250MVA and the base MVA 0.1 MVA. Comparisons of Figures 5 and 7 and Tables 6 and 7, the effects with and without substation bus for harmonic analysis can be clearly observed. From the simple test cases, it can be seen that the substation bus can be integrated into the proposed method without difficulty.

Table 7. Voltage THD (\%) of a simple seven-bus test system with substation bus.

\begin{tabular}{cccc}
\hline \multirow{2}{*}{ Bus } & & Voltage THD (\%) & \\
\cline { 2 - 4 } & Phase $\boldsymbol{a}$ & Phase $\boldsymbol{b}$ & Phase $\boldsymbol{c}$ \\
\hline 1 & 0.6138 & 0.5071 & 0.3235 \\
2 & 2.1326 & 1.9484 & 1.4811 \\
3 & 5.0356 & 6.384 & 4.3113 \\
4 & 5.0490 & - & - \\
5 & 5.0494 & 2.9622 & 3.1272 \\
6 & 3.7258 & 2.5141 & 2.6094 \\
7 & - & 2.5693 & 2.6155 \\
\hline
\end{tabular}




\subsection{Feasibility and Performance Tests}

An actual three-phase distribution system, including 6 laterals, 105 segments and 123 load points in the suburban area [21], is also used to test the proposed method. Different test scenarios have been used to demonstrate the feasibility of the proposed method; however, only one test scenario is shown here. Figure 8 illustrates that ten three-phase nonlinear loads and five three-phase harmonic filters are interconnected to the actual distribution system. The harmonic currents for the $5^{\text {th }}$-order harmonic order are also illustrated in Figure 8. Obviously, the harmonic currents absorbed by the harmonic filters can be effectively calculated.

Figure 8. Harmonic currents of the $5^{\text {th }}$-order harmonic order.

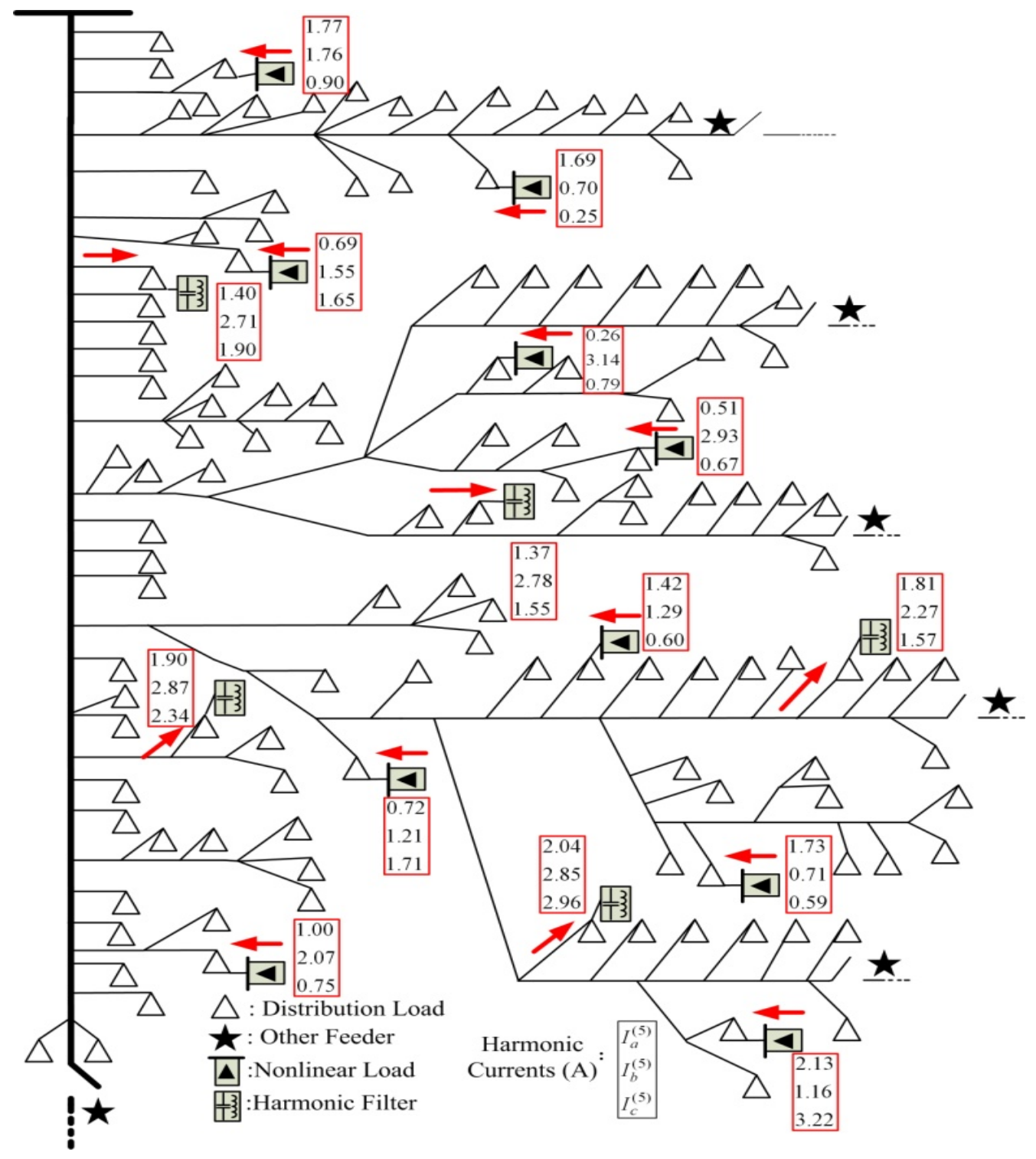

The voltage THD (\%) for each harmonic load and harmonic filter is illustrated in Figure 9. Note that the number of harmonic filters is 15 , because five three-phase harmonic filters are installed in the actual distribution system. Only the $\left[\mathbf{Z H A}^{(h)}\right]$ matrix with a dimension of 15 needs to be inverted; therefore, the proposed method has better computational performance and has the feasibility to be used in actual distribution systems. 
In order to demonstrate the computational performance of the proposed method, two other methods are used for comparisons. The three methods include:

Method 1: the proposed method;

Method 2: the commonly-used admittance-matrix-based method [10] and;

Method 3: the forward/backward sweep based method [16].

Figure 9. Voltage THD (\%).

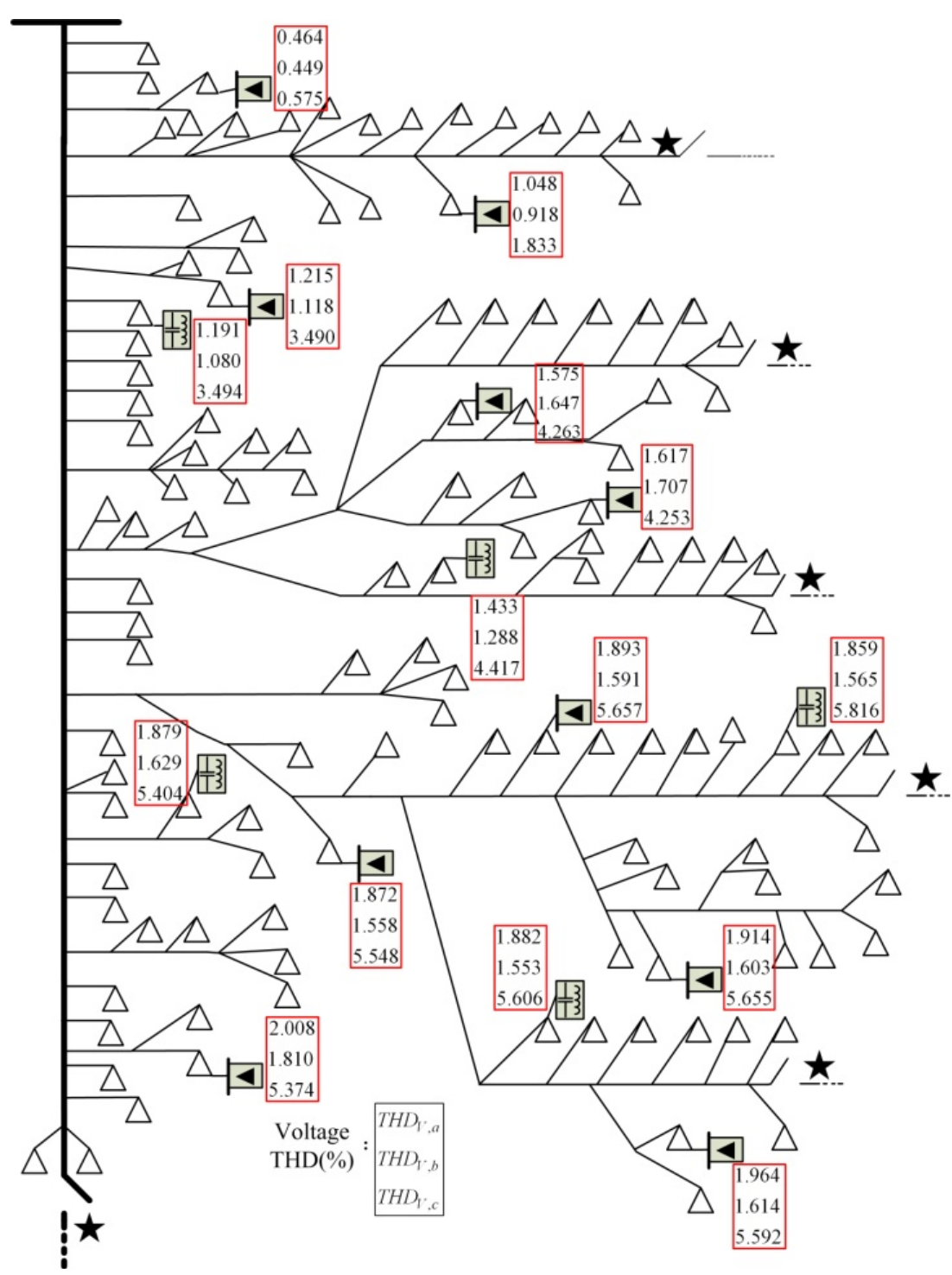

In order to demonstrate the computational performance of the proposed method, four three-phase systems, 15-, 32-, 63-, and 88-bus systems represented in [16], are used for our tests. Table 8 shows the normalized execution time (NET) of these three methods. From Table 8, it can be observed that the NETs of 88-bus system for Methods 1 and 2 are 17.53 and 3020.91 (about 172 times faster), respectively. Since the full admittance matrix inverse for each harmonic order can be omitted, Method 1 outperforms Method 2 for large-scale systems. The computational performances for Methods 1 and 3 are close. The NETs as shown in Table 8 include the computational time for load flow solution. The computational time of the forward/backward sweep based load flow method is less; therefore, the performance of Method 3 is better than Method 1. 
Table 8. Performance comparisons.

\begin{tabular}{ccccccc}
\hline Test case & Bus number & $\boldsymbol{N f}^{+}$ & $\boldsymbol{N s}^{+}$ & Method 1 & Method 2 & Method 3 \\
\hline 1 & 15 & 2 & 15 & $1 *$ & 12.09 & 0.92 \\
2 & 32 & 3 & 30 & 2.77 & 192.42 & 1.87 \\
3 & 63 & 5 & 30 & 9.14 & 920.62 & 5.23 \\
4 & 88 & 6 & 36 & 17.53 & 3020.91 & 9.48 \\
\hline
\end{tabular}

$*$ : NET 1.0 is set for the 15 -bus system in Method $1 ;{ }^{+} N s$ and $N f$ are the numbers of nonlinear loads and shunt equipments, respectively.

The NETs for Methods 1 and 3 excluding the computational time for a load flow solution are shown in Table 9. Table 9 shows that, excluding the computational time of load flow, Method 1 is slightly faster than Method 3. Although, the performance differences are not very obvious, the proposed method provides novel viewpoints in observing the branch currents and bus voltages under harmonic pollution in the solution procedures. Besides, Reference [12] needs the specific data format such as the coefficient vector defined to record whether the system harmonic currents flow through the branch to be designed and built first. The specific data format is then integrated into the commonly used forward/backward sweep techniques to calculate harmonic propagation. Therefore, the method proposed in [12] cannot be straightforwardly used to solve other harmonic problems, i.e., optimal harmonic filter design, etc. No specific data format needs to be designed and integrated in the solution procedure of Method 1. Moreover, the novel viewpoints in observing the branch currents and bus voltages under harmonic pollution which are vital for harmonic filter design can also be obtained by in the solution procedures of the proposed method. Therefore, the effectiveness and efficiency of the proposed method can be demonstrated.

Table 9. Performance comparisons for Methods 1 and 3 excluding the computational time of load flow.

\begin{tabular}{cccccc}
\hline Test case & Bus number & $\boldsymbol{N} \boldsymbol{f}$ & $\boldsymbol{N s}$ & Method 1 & Method 3 \\
\hline 1 & 15 & 2 & 15 & $1 *$ & 1.05 \\
2 & 32 & 3 & 30 & 1.34 & 1.56 \\
3 & 63 & 5 & 30 & 1.85 & 2.14 \\
4 & 88 & 6 & 36 & 2.31 & 2.73 \\
\hline
\end{tabular}

*: NET 1.0 is set for the 15-bus system in Method 1.

The phase information of the harmonic current sources can enhance the accuracy of harmonic analysis. The phase information for the different harmonic current sources in a distribution system needs a reference angle and the conventional power quality analyzer cannot determine the reference angle. Phasor-measurement unit can be used to acquire the phase information [22]; however, it is too expensive to be used in distribution systems. If the phase information of the harmonic current sources is acquired, the harmonic currents with phase information can be integrated into the proposed harmonic analysis method without modifying the proposed method. Without the phase information of the harmonic current sources in the above tests, the results can be considered as worst cases for harmonic analysis. 


\section{Conclusions}

Two relationship matrices derived from the topological characteristics of the distribution systems were used to analyze the variations in bus voltages, bus current injections and branch currents affected by harmonic current injections. Accordingly, some useful formulas were derived to analyze the three-phase harmonic propagation problem. The proposed method can provide novel viewpoints in calculating the branch currents and bus voltages under harmonic pollution. The harmonic currents aborted by the shunt equipments can also be easily quantified in the proposed solution procedure. This data is vital for harmonic filter design. Several tests were conducted to demonstrate the performance and feasibility of the proposed method. Moreover, the proposed method can obtain the same solutions as those obtained by the commonly-used methods with less computational time; therefore, the proposed method has great potential to be used in on-line operation. The applications of the proposed method for optimal harmonic filter design and harmonic resonance mitigation will be discussed in future works.

\section{Acknowledgments}

This work was supported in part by the National Science Council (NSC) of Taiwan under Grant NSC 101-2628-E-110-004-MY3 and NSC 102-3113-P-110-007.

\section{Conflicts of Interest}

The authors declare no conflict of interest.

\section{Appendix}

The relationship matrices, BIBC and BCBV in Equations (4) and (5), for Figure 3 can be built according to the building algorithms proposed in $[17,18]$. The non-zero terms of these two matrices are expressed in Equations (A1a) and (A1b), respectively. The vectors of BIBC and BCBV matrices relating to the harmonic currents $I s^{(h)}$ and the harmonic currents $I f^{(h)}$ are also marked in Equation (A1). The matrices as expressed in Equation (A2) relating to Equations (16) and (17) can then be built to calculate the $h^{\text {th }}$-order harmonic currents absorbed by the harmonic filters. The $h^{\text {th }}$-order harmonic propagation including the effects of harmonic currents on branch currents and bus voltages can be calculated by Equations (9) and (11) accordingly:

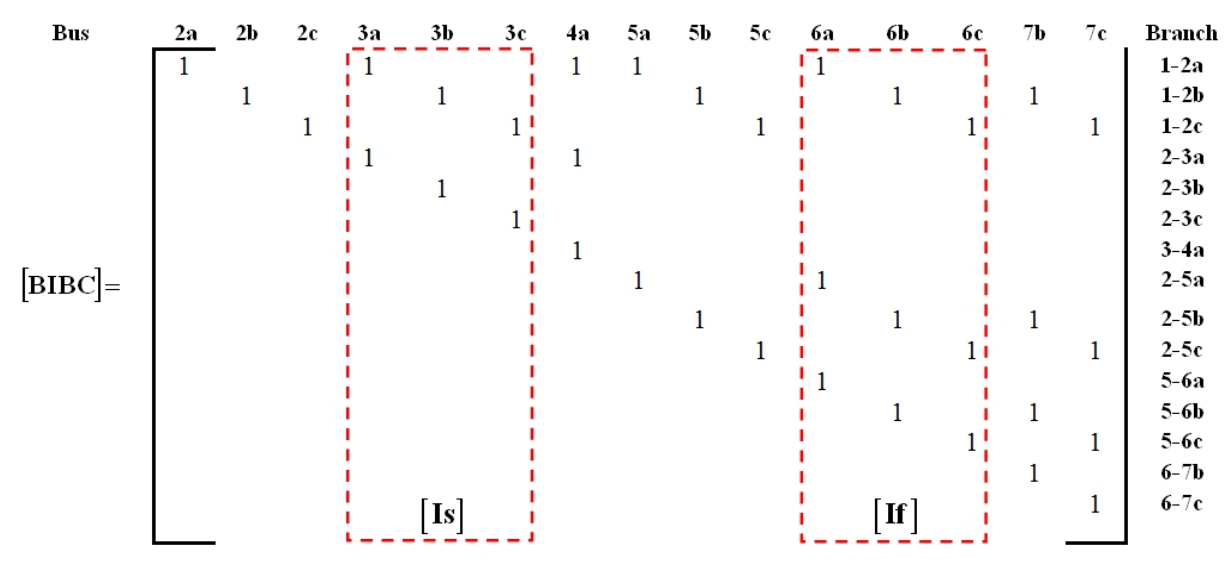




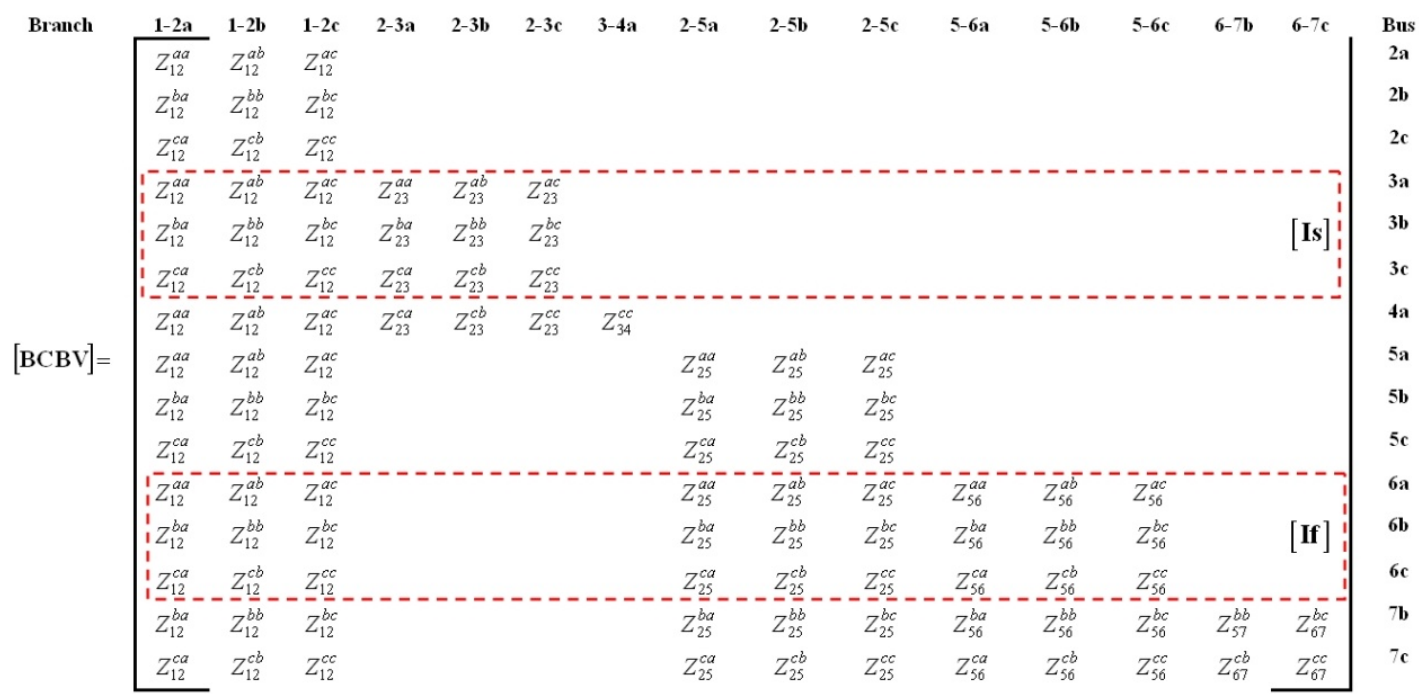

(A1b)

$$
[\mathbf{Z F}]=\left[\begin{array}{ccc}
Z f_{6 a}^{(h)} & 0 & 0 \\
0 & Z f_{6 b}^{(h)} & 0 \\
0 & 0 & Z f_{6 c}^{(h)}
\end{array}\right]
$$

$$
\left[\begin{array}{l}
\mathbf{B I B C}_{3 a} \\
\mathbf{B I B C}_{3 b} \\
\mathbf{B I B C}_{3 c}
\end{array}\right]^{T}=\left[\begin{array}{lllllllllllllll}
1 & 0 & 0 & 1 & 0 & 0 & 0 & 0 & 0 & 0 & 0 & 0 & 0 & 0 & 0 \\
0 & 1 & 0 & 0 & 1 & 0 & 0 & 0 & 0 & 0 & 0 & 0 & 0 & 0 & 0 \\
0 & 0 & 1 & 0 & 0 & 1 & 0 & 0 & 0 & 0 & 0 & 0 & 0 & 0 & 0
\end{array}\right]^{T}
$$

$$
\left[\begin{array}{l}
\mathbf{B I B C}_{6 a} \\
\mathbf{B I B C}_{6 b} \\
\mathbf{B I B C}_{6 c}
\end{array}\right]^{T}=\left[\begin{array}{lllllllllllllll}
1 & 0 & 0 & 0 & 0 & 0 & 0 & 1 & 0 & 0 & 1 & 0 & 0 & 0 & 0 \\
0 & 1 & 0 & 0 & 0 & 0 & 0 & 0 & 1 & 0 & 0 & 1 & 0 & 0 & 0 \\
0 & 0 & 1 & 0 & 0 & 0 & 0 & 0 & 0 & 1 & 0 & 0 & 1 & 0 & 0
\end{array}\right]^{T}
$$

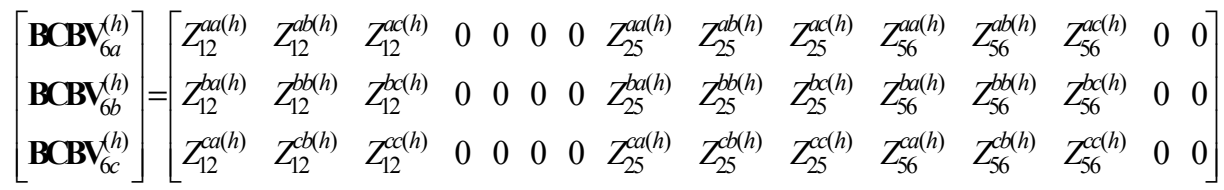

$$
[\mathbf{Z I S}]=\left[\begin{array}{l}
\mathbf{B C B V}_{6 a}^{(h)} \\
\mathbf{B C B V}_{6 b}^{(h)} \\
\mathbf{B C B V}_{6 c}^{(h)}
\end{array}\right]\left[\begin{array}{l}
\mathbf{B I B C}_{3 a} \\
\mathbf{B I B C}_{3 b} \\
\mathbf{B I B C}_{3 c}
\end{array}\right]^{T}=\left[\begin{array}{ccc}
Z_{12}^{a a(h)} & Z_{12}^{a b(h)} & Z_{12}^{a c(h)} \\
Z_{12}^{b a(h)} & Z_{12}^{b b(h)} & Z_{12}^{b c(h)} \\
Z_{12}^{c a(h)} & Z_{12}^{c b(h)} & Z_{12}^{c c(h)}
\end{array}\right]
$$

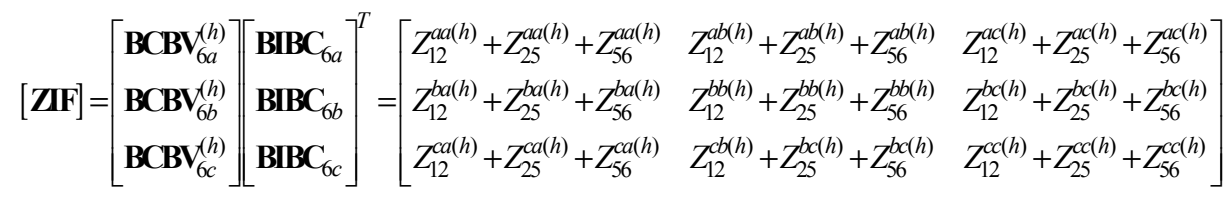

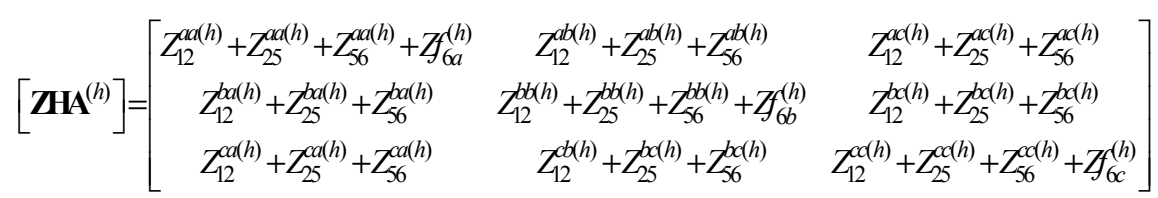




\section{References}

1. Wagner, V.E.; Balda, J.C.; Griffith, D.C.; McEachern, A.; Barnes, T.M.; Hartmann, D.P.; Phileggi, D.J.; Emannuel, A.E.; Horton, W.F.; Reid, W.E.; et al. Effects of the harmonics on equipment. IEEE Trans. Power Deliv. 1994, 8, 672-680.

2. Task Force on Harmonics Modeling and Simulation. Modeling and simulation of the propagation of harmonics in electric power network. I. Concepts, models and simulation techniques. IEEE Trans. Power Deliv. 1996, 11, 452-465.

3. Task Force on Harmonics Modeling and Simulation. Modeling and simulation of the propagation of harmonics in electric power network. II. Sample systems and examples. IEEE Trans. Power Deliv. 1996, 11, 466-474.

4. Herraiz, S.; Sainz, L.; Clua, J. Review of harmonic load flow formulations. IEEE Trans. Power Deliv. 2003, 18, 1079-1087.

5. Zheng, T.; Makram, E.B.; Girgis, A.A. Power system transient and harmonic studies using wavelet transform. IEEE Trans. Power Deliv. 1999, 14, 1461-1468.

6. Yan, Y.H.; Chen, C.S.; Moo, C.S.; Hsu, C.T. Harmonic analysis for industrial customers. IEEE Trans. Ind. Appl. 1994, 30, 462-468.

7. Arrillaga, J.; Callaghan, C.D. Three phase AC-DC load and harmonic flows. IEEE Trans. Power Deliv. 1991, 6, 238-244.

8. Xu, W.; Marti, J.R.; Dommel, H.W. A multiphase harmonic load flow solution technique. IEEE Trans. Power Syst. 1991, 6, 174-182.

9. Hong, Y.-Y.; Chen, Y.-T. Three-phase optimal harmonic power flow. IEEE Proc. Gener. Transm. Distrib. 1996, 143, 321-328.

10. De Saa, M.A.M.L.; Garcia, J.U. Three-phase harmonic load flow in frequency and time domains. IEEE Proc. Electr. Power Appl. 2003, 150, 295-300.

11. Lin, W.H.; Zhan, T.S.; Tsay, M.T. Multiple-frequency three-phase load flow for harmonic analysis. IEEE Trans. Power Syst. 2004, 19, 897-904.

12. Teng, J.H.; Chang, C.Y. Backward/forward sweep based harmonic analysis method for distribution systems. IEEE Trans. Power Deliv. 2007, 22, 1665-1672.

13. Ladjavardi, M.; Masoum, M.A.S. Genetically optimized fuzzy placement and sizing of capacitor banks in distorted distribution networks. IEEE Trans. Power Deliv. 2008, 23, 449-456.

14. De Arruda, E.F.; Kagan, N.; Ribeiro, P.F. Harmonic distortion state estimation using an evolutionary strategy. IEEE Trans. Power Deliv. 2010, 25, 831-842.

15. Romero, A.A.; Zini, H.C.; Ratta, G.; Dib, R. Harmonic load-flow approach based on the possibility theory. IET Gener. Transm. Distrib. 2011, 5, 393-404.

16. Eajal, A.A.; El-Hawary, M.E. Optimal capacitor placement and sizing in unbalanced distribution systems with harmonics consideration using particle swarm optimization. IEEE Trans. Power Deliv. 2010, 25, 1734-1741.

17. Teng, J.H. A direct approach for distribution system load flow solutions. IEEE Trans. Power Deliv. 2003, $18,882-887$.

18. Teng, J.H. Unsymmetrical short-circuit fault analysis for weakly-meshed distribution systems. IEEE Trans. Power Syst. 2010, 25, 96-105. 
19. Arrilliaga, J.; Bradley, D.A.; Bodger, P.S. Power System Harmonics; John Wiley and Sons: New York, NY, USA, 1985.

20. Grainger, J.J.; Stevenson, W.D. Power System Analysis; McGraw-Hill International Editions: New York, NY, USA, 1994.

21. Ho, C.Y.; Lee, T.E.; Lin, C.H. Optimal placement of fault indicators using immune algorithm. IEEE Trans. Power Syst. 2011, 26, 38-45.

22. Yu, C.S.; Liu, C.W.; Yu, S.L.; Jiang, J.A. A new PMU-based fault location algorithm for series compensated lines. IEEE Trans. Power Deliv. 2002, 17, 33-46.

(C) 2014 by the authors; licensee MDPI, Basel, Switzerland. This article is an open access article distributed under the terms and conditions of the Creative Commons Attribution license (http://creativecommons.org/licenses/by/3.0/). 